\title{
Management of brain metastases from renal cell carcinoma
}

\section{Suguru Shirotake}

Department of Uro-Oncology, Saitama Medical University International Medical Center, Saitama, Japan

Correspondence to: Suguru Shirotake, MD, PhD. Department of Uro-Oncology, Saitama Medical University International Medical Center, 1397-1 Yamane, Hidaka, Saitama 350-1298, Japan. Email: ss6001@5931.saitama-med.ac.jp.

Provenance: This is an invited article commissioned by the Section Editor Dr. Xiao Li, MD (Department of Urology, Jiangsu Cancer Hospital \& Jiangsu Institute of Cancer Research \& Nanjing Medical University Affiliated Cancer Hospital, Nanjing, China).

Comment on: Flippot R, Dalban C, Laguerre B, et al. Safety and Efficacy of Nivolumab in Brain Metastases From Renal Cell Carcinoma: Results of the GETUG-AFU 26 NIVOREN Multicenter Phase II Study. J Clin Oncol 2019;37:2008-16.

Submitted Aug 19, 2019. Accepted for publication Aug 28, 2019.

doi: 10.21037/atm.2019.08.111

View this article at: http://dx.doi.org/10.21037/atm.2019.08.111

The most common primary tumors associated with brain metastases are lung (20-56\%), melanoma (6-11\%), breast $(5-30 \%)$, and renal cell carcinoma (RCC) $(2-7 \%)(1,2)$. However, the true incidence is likely higher than these estimates because most guidelines for solid tumors do not recommend routine brain magnetic resonance imaging (MRI) screening in patients without neurological symptoms. Recent clinical trial enrolments often involve a concomitant requirement for brain MRI screening. Unfortunately, the safety and activity of immune checkpoint inhibitors (ICIs), which are already recommended for patients with metastatic clear cell RCC (mccRCC), have not been revealed in those with brain metastases because they were included in the exclusion criteria of crucial trials $(3,4)$. The current study, the GETUG-AFU 26 NIVOREN trial, prospectively assessed clinical outcome of nivolumab in mccRCC patients with asymptomatic brain metastases who progressed after molecular targeted therapies. This study considered two important issues, (I) brain imaging before ICIs treatment and (II) focal therapy prior to ICIs for asymptomatic brain metastases from RCC, which are discussed as below.

Various factors are linked with brain metastases including sex, age, ethnicity, tumor type, and molecular subtype. In the Metropolitan Detroit Cancer Surveillance System, the incidence of brain metastases in lung cancer (21.4\% vs. $19.4 \%)$, melanoma (11.7\% vs. $7.1 \%)$ and breast cancer (7.4\% vs. $4.6 \%$ ) were higher in African Americans than other ethnic groups, however, the incidence of those in RCC was not (5.3\% vs. 6.8\%) (2). Focusing on the molecular signatures of brain metastases, the whole-exome sequencing of matched primary tumors (lung, breast, and RCCs) and brain metastases revealed that, although primary tumor and brain metastases clonally originated from a shared precursor, a diverse evolutionary pattern occurred at each site of metastases, suggesting that sequencing of primary biopsies alone may miss the opportunity for the optimal therapy (5). Although the tumor suppressor gene $V H L$ is well-known as a mutational driver for clear cell $\mathrm{RCC}$, brain metastases from RCC revealed mutations in the genes PTEN, CDKN2A, and PIK3CA that were not detected in the corresponding primary tumors (5). Above all, the present study showed that more than $10 \%$ of patients with mRCC had unexpectedly asymptomatic brain metastases before nivolumab treatment. Based on these findings that it is difficult to predict the presence of brain metastasis, we recommend performing brain imaging at the initial diagnosis of mRCC, even if the patient does not have neurological symptoms.

Radiographically, brain MRI irrespective of intravenous gadolinium contrast is recommended for the evaluation of brain metastases and has a higher sensitivity compared to contrast enhanced CT. Additionally, diffusion-weighted (DW)-MRI, which differentiates abscesses or high-grade gliomas, and fluid-attenuated inversion recovery (FLAIR) MRI, which shows vasogenic edema as areas of increased signal intensity, can be helpful in the clinical evaluation of brain metastases (6). ${ }^{18} \mathrm{~F}$-fluorodeoxyglucose (FDG)-PET may provide sufficient information to differentiate between brain metastases and lymphoma, but not glioma, when differential diagnoses are difficult using MRI alone (7). 
In the context of histopathology, as well as management, surgical treatment for brain metastases should be deliberated in patients with immediate neurological symptoms at initial diagnosis in whom the primary tumor has not yet been identified, and also in patients with two or three brain metastases, high performance status and wellcontrolled systemic disease (8). Cerebrospinal fluid (CSF) examination has not been routinely performed for the histopathological diagnosis of brain metastases; however, next-generation sequencing of CSF has recently revealed some crucial genetic alterations in brain metastases, such as an $E G F R$ mutation in non-small cell lung cancer, a $B R A F$ mutation in melanoma and a HER2 amplification in breast cancer, but unfortunately not yet in RCC (9). Further studies are needed to validate liquid biopsies and investigate tumor biology in the CNS.

The aims of treating brain metastases can be the relief of neurological symptoms and tumor control. Surgery, radiotherapy, and/or systemic medical therapies are generally combined to maximize the quality of life and prolong overall survival. Immunotherapies as novel treatments for brain metastases, including anti-PD-1/PDL1 and anti-CTLA-4 antibodies, as well as combinations of these antibodies, have been reported in patients with nonsmall cell lung cancer and melanoma (10). Although the general agreement is that monoclonal antibodies cannot penetrate the central CNS, the intracranial effects of ICIs could be due to dual mechanistic pharmacodynamic actions: (I) they can bind PD-1 or CTLA-4 irreversibly on lymphocytes that can penetrate the blood brain barrier (BBB); and (II) these antibodies can pass the BBB and inhibit the tumor infiltrating lymphocytes within the intracranial tumor (11). Also, using a murine model with melanoma brain metastases, the efficacy of ICIs appeared to be influenced by the presence of extracranial tumors; it is hypothesized that a synergy between ICIs and extracranial tumors enhances the recruitment of cytotoxic $\mathrm{T}$ cells to the brain through peripheral development of effector $\mathrm{T}$ cells and upregulation of $\mathrm{T}$ cell receptors on tumor blood vessels (12).

The prognosis of patients with melanoma who have brain metastases has remained extremely poor, with a median overall survival of 4 to 5 months in the pre-immunotherapy era. In a recent phase II trial, combined nivolumab and ipilimumab in melanoma patients who had untreated brain metastases, showed a high intracranial response rate $(57 \%$, including $26 \%$ of patients with complete response), as well as extracranial clinical benefit (56\%). Overall survival was $81.5 \%$ at 1 year and more than $70 \%$ at 2 years (13). Radiotherapy, such as whole-brain radiation therapy (WBRT) and stereotactic radiosurgery (SRS), with or without surgery, plays a critical role in the management of brain metastases with neurological symptoms. Based on small retrospective studies, combined regimens of these radiotherapies with immunotherapies have also been explored $(14,15)$. Interestingly, the present prospective study showed the clinical benefit of focal brain therapy prior to ICIs even in mRCC patients with asymptomatic brain metastases. Further studies will be needed to assess long-term outcomes with respect to intra- and extracranial disease control and late toxicity due to the synergism of combined treatment.

\section{Acknowledgments}

None.

\section{Footnote}

Conflicts of Interest: The author has no conflicts of interest to declare.

Ethical Statement: The author is accountable for all aspects of the work in ensuring that questions related to the accuracy or integrity of any part of the work are appropriately investigated and resolved.

\section{References}

1. Nayak L, Lee EQ, Wen PY. Epidemiology of brain metastases. Curr Oncol Rep 2012;14:48-54.

2. Barnholtz-Sloan JS, Sloan AE, Davis FG, et al. Incidence proportions of brain metastases in patients diagnosed (1973 to 2001) in the Metropolitan Detroit Cancer Surveillance System. J Clin Oncol 2004;22:2865-72.

3. Motzer RJ, Escudier B, McDermott DF, et al. Nivolumab versus Everolimus in Advanced Renal-Cell Carcinoma. N Engl J Med 2015;373:1803-13.

4. Motzer RJ, Tannir NM, McDermott DF, et al. Nivolumab plus Ipilimumab versus Sunitinib in Advanced Renal-Cell Carcinoma. N Engl J Med 2018;378:1277-90.

5. Brastianos PK, Carter SL, Santagata S, et al. Genomic Characterization of Brain Metastases Reveals Branched Evolution and Potential Therapeutic Targets. Cancer Discov 2015;5:1164-77.

6. Achrol AS, Rennert RC, Anders C, et al. Brain metastases. 
Nat Rev Dis Primers 2019;5:5.

7. Kosaka N, Tsuchida T, Uematsu H, et al. 18F-FDG PET of common enhancing malignant brain tumors. AJR Am J Roentgenol 2008;190:W365-9.

8. Soffietti R, Abacioglu U, Baumert B, et al. Diagnosis and treatment of brain metastases from solid tumors: guidelines from the European Association of NeuroOncology (EANO). Neuro Oncol 2017;19:162-74.

9. Pentsova EI, Shah RH, Tang J, et al. Evaluating Cancer of the Central Nervous System Through Next-Generation Sequencing of Cerebrospinal Fluid. J Clin Oncol 2016;34:2404-15.

10. Berghoff AS, Venur VA, Preusser M, et al. Immune Checkpoint Inhibitors in Brain Metastases: From Biology to Treatment. Am Soc Clin Oncol Educ Book 2016;35:e116-22.

11. van Bussel MTJ, Beijnen JH, Brandsma D. Intracranial antitumor responses of nivolumab and ipilimumab: a

Cite this article as: Shirotake S. Management of brain metastases from renal cell carcinoma. Ann Transl Med 2019;7(Suppl 8):S369. doi: 10.21037/atm.2019.08.111 pharmacodynamic and pharmacokinetic perspective, a scoping systematic review. BMC Cancer 2019;19:519.

12. Taggart D, Andreou T, Scott KJ, et al. Anti-PD-1/antiCTLA-4 efficacy in melanoma brain metastases depends on extracranial disease and augmentation of CD8(+) T cell trafficking. Proc Natl Acad Sci U S A 2018;115:E1540-9.

13. Tawbi HA, Forsyth PA, Algazi A, et al. Combined Nivolumab and Ipilimumab in Melanoma Metastatic to the Brain. N Engl J Med 2018;379:722-30.

14. Anderson ES, Postow MA, Wolchok JD, et al. Melanoma brain metastases treated with stereotactic radiosurgery and concurrent pembrolizumab display marked regression; efficacy and safety of combined treatment. J Immunother Cancer 2017;5:76.

15. Nardin C, Mateus C, Texier M, et al. Tolerance and outcomes of stereotactic radiosurgery combined with antiprogrammed cell death-1 (pembrolizumab) for melanoma brain metastases. Melanoma Res 2018;28:111-9. 